T. Kaneyama

Nagoya Math. J.

Vol. 111 (1988), 25-40

\title{
TORUS-EQUIVARIANT VECTOR BUNDLES ON PROJECTIVE SPACES
}

\author{
TAMAFUMI KANEYAMA
}

\section{Introduction}

For a free $Z$-module $N$ of rank $n$, let $T=T_{N}$ be an $n$-dimensional algebraic torus over an algebraically closed field $k$ defined by $N$. Let $X=T_{N}$ emb ( $\left.\Delta\right)$ be a smooth complete toric variety defined by a fan $\Delta$ (cf. [6]). Then $T$ acts algebraically on $X$. A vector bundle $E$ on $X$ is said to be an equivariant vector bundle, if there exists an isomorphism $f_{t}: t^{*} E \rightarrow E$ for each $k$-rational point $t$ in $T$, where $t: X \rightarrow X$ is the action of $t$. Equivariant vector bundles have $T$-linearizations (see Definition 1.2 and [2], [4]), hence we consider $T$-linearized vector bundles.

The $n$-dimensional projective space $\boldsymbol{P}^{n}$ has a natural action of $T$ and can be regarded as a toric variety. In [4], we classified indecomposable equivariant vector bundles of rank two on $\boldsymbol{P}^{2}$. When $n>2$, Hartshorne [3] constructed vector bundles of rank two from codimension two subschemes satisfying certain conditions. Bertin and Elencwajg [2] then used this method to construct equivariant vector bundles of rank two on $\boldsymbol{P}^{n}$ and showed that there exist no indecomposable equivariant vector bundles of rank two on $\boldsymbol{P}^{n}$ which are obtained in this way.

In this paper, we generalize our method in [4] to show that there exist no indecomposable equivariant vector bundles of rank $r(1<r<n)$ on $\boldsymbol{P}^{n}$ (Corollary 3.5) and that indecomposable equivariant vector bundles of rank $n$ on $\boldsymbol{P}^{n}$ are isomorphic to $E(d)$ or $E^{*}(d)$ for some integer $d$, where $E$ is defined by an exact sequence

$$
0 \longrightarrow \mathcal{O}_{\boldsymbol{P}^{n}} \longrightarrow \bigoplus_{i=0}^{n} \mathcal{O}_{\boldsymbol{P}^{n}}\left(a_{i}\right) \longrightarrow E \longrightarrow 0
$$

for positive integers $a_{i}$.

Received August 23, 1986.

Revised April 28, 1987. 


\section{§1. Preliminaries}

Let $N$ be a free $Z$-module of rank $n$. Let $M$ be the dual $Z$-module of $N$. Then there is a natural $Z$-bilinear map

$$
\langle,\rangle: M \times N \longrightarrow Z \text {. }
$$

It can naturally be extended to $M_{R} \times N_{R} \rightarrow R$, where $M_{R}=M \otimes_{Z} R$ and $N_{R}=N \otimes_{z} R$. We denote $\varphi(\xi)=\langle\xi, \varphi\rangle$ for $\xi$ in $M_{R}$ and $\varphi$ in $N_{R}$. Let $T=T_{N}$ be an $n$-dimensional algebraic torus defined by $N$ over an algebraically closed field $k$. Then we can identify $M$ with the additive group of characters of $T$. Let $X=T_{N} \mathrm{emb}(\Delta)$ be a smooth complete toric variety of dimension $n$ defined by a fan $\Delta$ of $N$ for which the reader is referred to [6].

Definition 1.1. An equivariant vector bundle $E$ on $X$ is a vector bundle on $X$ such that there exists an isomorphism $f_{t}: t^{*} E \rightarrow E$ for every $k$-rational point $t$ in $T$, where $t: X \rightarrow X$ is the action of $t$ on $X$.

Definition 1.2. An equivariant vector bundle $E=\left(E, f_{t}\right)$ is said to be $T$-linearized if $f_{t t^{\prime}}=f_{t^{\prime}} \circ t^{\prime} *\left(f_{t}\right)$ holds for every pair of $k$-rational points $t, t^{\prime}$ of $T$, where

$$
f_{t t^{\prime}}=f_{t^{\prime}} \circ t^{\prime *}\left(f_{t}\right):\left(t t^{\prime}\right)^{*} E \stackrel{t^{\prime *}\left(f_{t}\right)}{\longrightarrow} t^{\prime *} E \stackrel{f_{t^{\prime}}}{\longrightarrow} E .
$$

In [4], we showed that an equivariant vector bundle necessarily has a $T$-linearization. We also studied how to describe $T$-linearized vector bundles in terms of fans, as we now recall.

We denote by $\Delta(l)$ the set of $l$-dimensional cones in $\Delta$. For $C$ in $\Delta(l)$, there exists a finite subset $\left\{\varphi_{1}, \cdots, \varphi_{l}\right\}$ of $N$ such that $C=\boldsymbol{R}_{0} \varphi_{1}+\cdots$ $+\boldsymbol{R}_{0} \varphi_{l}$, where $\boldsymbol{R}_{0}$ is the set of non-negative real numbers. We say that $\left\{\varphi_{1}, \cdots, \varphi_{l}\right\}$ is the fundamental system of generators of $C$ if $\varphi_{i}$ are primitive, i.e., $\varphi_{i}$ is not a non-trivial integral multiple of any element of $N$. The fundamental system of generators $\left\{\varphi_{1}, \cdots \varphi_{l}\right\}$ of $C$ is uniquely determined by $C$ and is denoted by $|C|$. We consider the following:

( I ) $m:\left\{\left|C^{\prime}\right| \mid C^{\prime} \in \Delta(1)\right\} \longrightarrow Z^{\oplus r}$

sending $\varphi$ to $m(\varphi)=\left(m(\varphi)_{1}, \cdots, m(\varphi)_{r}\right)$, and for every $C$ in $\Delta(n)$

$$
m_{C}:|C| \longrightarrow Z^{\oplus r}
$$

so that there exists a permutation $\tau=\tau_{C}$ such that

$$
m_{C}(\varphi)=\left(m_{C}(\varphi)_{1}, \cdots, m_{C}(\varphi)_{r}\right)=\left(m(\varphi)_{\tau(1)}, \cdots, m(\varphi)_{\tau(r)}\right)
$$


for every $\varphi$ in $|C|$.

Let $C$ be an $n$-dimensional cone in $\Delta(n)$. Then we have a set of characters $\left\{\xi(C)_{1}, \cdots, \xi(C)_{r}\right\}$ in $M$ by solving, for each $1 \leq i \leq r$, the equations $\varphi\left(\xi(C)_{i}\right)=m_{C}(\varphi)_{i}$ for every $\varphi$ in $|C|$. Then it is easy to see that (I) is equivalent to the following:

( I') $\xi: \Delta(n) \longrightarrow M^{\oplus r}$

sending $C$ to $\xi(C)=\left(\xi(C)_{1}, \cdots, \xi(C)_{r}\right)$ such that there exists a permutation $\tau=\tau_{C, C^{\prime}}$ for every pair of cones $C$ and $C^{\prime}$ in $\Delta(n)$, so that $\varphi\left(\xi(C)_{i}\right)=$ $\varphi\left(\xi\left(C^{\prime}\right)_{\tau(i)}\right)$ for every $i$ and every $\varphi$ in $|C| \cap\left|C^{\prime}\right|$.

(II) $P: \Delta(n) \times \Delta(n) \longrightarrow G L_{r}(k)$

sending $\left(C, C^{\prime}\right)$ to $P\left(C, C^{\prime}\right)=\left(P\left(C, C^{\prime}\right)_{i j}\right)$ such that $P\left(C, C^{\prime}\right)_{i j} \neq 0$ only if $\varphi\left(\xi(C)_{i}\right) \geq \varphi\left(\xi\left(C^{\prime}\right)_{j}\right)$ for every $\varphi$ in $|C| \cap\left|C^{\prime}\right|$ and that

$$
P\left(C, C^{\prime}\right) P\left(C^{\prime}, C^{\prime \prime}\right)=P\left(C, C^{\prime \prime}\right)
$$

for every $C, C^{\prime}, C^{\prime \prime}$ in $\Delta(n)$.

For $(m, P)$ defined by $(\mathrm{I})$ and (II), we denote by $E(m, P)$ the $T$-linearized vector bundle obtained from $(m, P)$. We refer the reader to [4] as for the construction of the $T$-linearized vector bundle $E(m, P)$.

(III) Two pairs $(m, P)$ and $\left(m^{\prime}, P^{\prime}\right)$ defined by (I) and (II) are said to be equivalent if there exists a permutation $\tau=\tau_{C}$ for every $C$ in $\Delta(n)$ such that

$$
\left(m_{c}(\varphi)_{1}, \cdots, m_{C}(\varphi)_{r}\right)=\left(m_{C}^{\prime}(\varphi)_{\tau(1)}, \cdots, m_{C}^{\prime}(\varphi)_{\tau(r)}\right)
$$

for every $\varphi$ in $|C|$ and if there exists

$$
\sigma: \Delta(n) \longrightarrow G L_{r}(k)
$$

such that $\sigma(C)_{i j} \neq 0$ only if $\varphi\left(\xi(C)_{i}\right) \geq \varphi\left(\xi(C)_{\jmath}\right)$ for every $\varphi$ in $|C|$ and such that

$$
P^{\prime}\left(C, C^{\prime}\right)=\sigma(C)^{-1} P\left(C, C^{\prime}\right) \sigma\left(C^{\prime}\right)
$$

holds for every $C$ and $C^{\prime}$ in $\Delta(n)$.

TheOREM 1.3 (cf. ([4]). Let $X=T_{N}$ emb ( $(\Delta)$ be a smooth complete toric variety defined by a fan 4 . Then the set of T-linearized vector bundles of rank $r$ up to T-isomorphism corresponds bijectively to the set of (I) (or ( $\left.\mathrm{I}^{\prime}\right)$ ) and (II) up to the equivalence (III).

Remark 1.4 Let $D_{\varphi}$ be the divisor corresponding to the con? $\boldsymbol{R}_{0} \varphi$ in $\Delta(1)$. Put $m_{\varphi}=m(\varphi)$ where $m$ is defined by (I) in the case $r=1$. Let 
$P\left(C, C^{\prime}\right)=1$ for every $C$ and $C^{\prime}$ in $\Delta(n)$. Then the $T$-linearized vector bundle $E(m, P)$ is the line bundle $\mathcal{O}_{X}\left(-\sum m_{\varphi} D_{\varphi}\right)$, where the summation is taken over $\varphi$ in $\{|C| \mid C \in \Delta(1)\}$.

Remark 1.5. Let $E=E(m, P)$ be the T-linearized vector bundle of rank $r$ defined by $(m, P)$. Then $E \otimes \mathcal{O}_{X}\left(-\sum m_{n} D_{\varphi}\right)$ is $T$-isomorphic to $E\left(m^{\prime}, P\right)$, where

$$
m^{\prime}(\varphi)=\left(m(\varphi)_{1}+m_{\varphi}, \cdots, m(\varphi)_{r}+m_{\varphi}\right)
$$

for every $\varphi$ in $\{|C| \mid C \in \Delta(1)\}$. The dual vector bundle $E^{*}$ is $T$-isomorphic to $E\left(-m,{ }^{t} P^{-1}\right)$, where

$$
{ }^{t} P^{-1}\left(C, C^{\prime}\right)={ }^{t} P\left(C, C^{\prime}\right)^{-1},
$$

and

$$
-m(\varphi)=\left(-m(\varphi)_{1}, \cdots,-m(\varphi)_{r}\right)
$$

for every $\varphi$ in $\{|C| \mid C \in \Delta(1)\}$.

\section{§. Some lemmas}

Lemma 2.1. Let $C$ and $C^{\prime}$ be two cones in $\Delta(n)$. Suppose $P\left(C, C^{\prime}\right)_{i i} \neq 0$ holds for every $i$. Then $\varphi\left(\xi(C)_{i}\right)=\varphi\left(\xi\left(C^{\prime}\right)_{i}\right)$ holds for every $i$ and every $\varphi$ in $|C| \cap\left|C^{\prime}\right|$.

Proof. Since $P\left(C, C^{\prime}\right)_{i i} \neq 0$ we have $\varphi\left(\xi(C)_{i}\right) \geq \varphi\left(\xi\left(C^{\prime}\right)_{i}\right)$ for every $i$ and every $\varphi$ in $|C| \cap\left|C^{\prime}\right|$. Hence

$$
\varphi\left(\xi(C)_{1}\right)+\cdots+\varphi\left(\xi(C)_{r}\right) \geq \varphi\left(\xi\left(C^{\prime}\right)_{1}\right)+\cdots+\varphi\left(\xi\left(C^{\prime}\right)_{r}\right) .
$$

Since the two sets $\left\{\varphi\left(\xi(C)_{1}\right), \cdots, \varphi\left(\xi(C)_{r}\right)\right\}$ and $\left\{\varphi\left(\xi\left(C^{\prime}\right)_{1}\right), \cdots, \varphi\left(\xi\left(C^{\prime}\right)_{r}\right)\right\}$ are the same sets by $\left(I^{\prime}\right)$, we have

$$
\varphi\left(\xi(C)_{1}\right)+\cdots+\varphi\left(\xi(C)_{r}\right)=\varphi\left(\xi\left(C^{\prime}\right)_{1}\right)+\cdots+\varphi\left(\xi\left(C^{\prime}\right)_{r}\right) .
$$

Therefore $\varphi\left(\xi(C)_{1}\right)=\varphi\left(\xi\left(C^{\prime}\right)_{1}\right), \cdots, \varphi\left(\xi(C)_{r}\right)=\varphi\left(\xi\left(C^{\prime}\right)_{r}\right)$ for every $\varphi$ in $|C| \cap\left|C^{\prime}\right|$.

Lemma 2.2. Let $C$ and $C^{\prime}$ be two cones in $\Delta(n)$ such that $C \cap C^{\prime}$ is in $\Delta(n-1)$. Then, by rearranging $\left\{\xi(C)_{1}, \cdots, \xi(C)_{r}\right\}$ and $\left\{\xi\left(C^{\prime}\right)_{1}, \cdots, \xi\left(C^{\prime}\right)_{r}\right\}$ and replacing $(m, P)$ by an equivalent pair, we can reduce the matrix $P\left(C, C^{\prime}\right)$ to an upper triangular matrix.

Proof. Put $P=P\left(C, C^{\prime}\right)$. Since $\operatorname{det}(P) \neq 0$, we may assume that $P_{i i} \neq 0$ for every $i$ by rearranging $\left\{\xi(C)_{i}\right\}$ and $\left\{\xi\left(C^{\prime}\right)_{i}\right\}$. Suppose that $P_{h k} \neq 0$ 
and $P_{k h}=0$ for some $h, k(h>k)$. Then, by interchanging $\xi(C)_{h}$ with $\xi(C)_{k}$ and $\xi\left(C^{\prime}\right)_{h}$ with $\xi\left(C^{\prime}\right)_{k}$ we have $P_{h k}=0, P_{k h} \neq 0$. So we may further assume that if $P_{h k} \neq 0$ for $h>k$ then $P_{k h} \neq 0$.

Suppose that $P$ is not an upper triangular matrix. Then we take minimal $k$ such that $P_{h k} \neq 0$ for some $h>k$. Then $P_{i j}=0$ for $j<k$ and $i>j$. Since $P_{h k} \neq 0$ and $P_{k h} \neq 0$ by assumption, we have

$$
\varphi\left(\xi(C)_{h}\right) \geq \varphi\left(\xi\left(C^{\prime}\right)_{k}\right) \text { and } \varphi\left(\xi(C)_{k}\right) \geq \varphi\left(\xi\left(C^{\prime}\right)_{h}\right)
$$

for every $\varphi$ in $|C| \cap\left|C^{\prime}\right|$. Consequently, for $\varphi$ in $|C| \cap\left|C^{\prime}\right|$, we have $\varphi\left(\xi(C)_{h}\right)$ $>\varphi\left(\xi\left(C^{\prime}\right)_{h}\right)$ if $\varphi\left(\xi(C)_{h}\right)>\varphi\left(\xi(C)_{k}\right)$, while $\varphi\left(\xi(C)_{k}\right)>\varphi\left(\xi\left(C^{\prime}\right)_{k}\right)$ if $\varphi\left(\xi(C)_{h}\right)<$ $\varphi\left(\xi(C)_{k}\right)$, a contradiction by Lemma 2.1. Therefore we have

$$
\varphi\left(\xi(C)_{h}\right)=\varphi\left(\xi(C)_{k}\right) \quad \text { for every } \varphi \text { in }|C| \cap\left|C^{\prime}\right| \text {. }
$$

Since $C \cap C^{\prime}$ is in $\Delta(n-1)$, put $|C|-|C| \cap\left|C^{\prime}\right|=\{\psi\}$. Suppose $\psi\left(\xi(C)_{h}\right)$ $<\psi\left(\xi(C)_{k}\right)$. Then we interchange $\xi(C)_{h}$ and $\xi(C)_{k}$. This procedure interchanges $P_{h i}$ with $P_{k i}$ for each $1 \leq i \leq r$. Therefore the minimality of $k$ is preserved. Hence we may assume that

$$
\varphi\left(\xi(C)_{h}\right) \geq \varphi\left(\xi(C)_{k}\right) \quad \text { for every } \varphi \text { in }|C| .
$$

Now we define $\sigma(C)=\left(\sigma(C)_{i j}\right)$ by

$$
\sigma(C)_{i j}= \begin{cases}1 & \text { for } i=j, \\ c \neq 0 & \text { for } i=h \text { and } j=k, \\ 0 & \text { otherwise, }\end{cases}
$$

and replace $(m, P)$ by an equivalent pair using this $\sigma(C)$. This is allowed by what we have just seen. In this way, we can reduce ourselves to the case $P_{h k}=0$. Hence we have $P_{i k}=0$ for all $i(i>k)$. After this replacement, however. $P_{i i}$ may be zero for $i>k$. By rearranging $\left\{\xi(C)_{k+1}\right.$, $\left.\cdots, \xi(C)_{r}\right\}$ and $\left\{\xi\left(C^{\prime}\right)_{k+1}, \cdots, \xi\left(C^{\prime}\right)_{r}\right\}$, we may assume that $P_{i i} \neq 0$ for every $i$. So we can repeat the same procedure, which will terminate after finitely many steps and leads to an upper triangular matrix $P$.

Lemma 2.3. Let $C, C^{\prime}, C^{\prime \prime}$ be three distinct cones in $\Delta(n)$ such that $C^{\prime} \cap C^{\prime \prime}$ is in $\Delta(n-1)$. Then, by rearranging $\left\{\xi(C)_{i}\right\},\left\{\xi\left(C^{\prime}\right)_{i}\right\}$ and $\left\{\xi\left(C^{\prime \prime}\right)_{i}\right\}$ and replacing $(m, P)$ by an equivalent pair, we can reduce ourselves to the situation where $P\left(C^{\prime}, C^{\prime \prime}\right)$ is an upper triangular matrix and $P\left(C, C^{\prime}\right)_{i i} \neq 0$ for every $i$. 
Proof. By Lemma 2.2, $P\left(C^{\prime}, C^{\prime \prime}\right)$ is first reduced to an upper triangular matrix. Since $\operatorname{det}\left(P\left(C, C^{\prime}\right)\right) \neq 0$, we have $P\left(C, C^{\prime}\right)_{i i} \neq 0$ by rearranging $\left\{\xi(C)_{1}, \cdots, \xi(C)_{r}\right\}$.

Corollary 2.4. Let $C, C^{\prime}, C^{\prime \prime}$ be three distinct cones in $\Delta(n)$ such that $C^{\prime} \cap C^{\prime \prime}$ is in $\Delta(n-1)$. Then by rearranging $\left\{\xi(C)_{i}\right\},\left\{\xi\left(C^{\prime}\right)_{i}\right\}$ and $\left\{\xi\left(C^{\prime \prime}\right)_{i}\right\}$ and replacing $(m, P)$ by an equivalent pair, we may assume that

$$
\varphi\left(\xi(C)_{i}\right)=\varphi\left(\xi\left(C^{\prime}\right)_{i}\right) \quad \text { for every } \varphi \text { in }|C| \cap\left|C^{\prime}\right|
$$

and

$$
\varphi\left(\xi\left(C^{\prime}\right)_{i}\right)=\varphi\left(\xi\left(C^{\prime \prime}\right)_{i}\right) \quad \text { for every } \varphi \text { in }\left|C^{\prime}\right| \cap\left|C^{\prime \prime}\right|
$$

hold for every $i$.

LEMMA 2.5. Let $C, C^{\prime}, C^{\prime \prime}$ be three distinct cones in $A(n)$ such that $C \cap C^{\prime}$ is in $A(n-1)$. Suppose that $P\left(C^{\prime}, C^{\prime \prime}\right)=I$ is the identity matrix. Then, by rearranging $\left\{\xi(C)_{i}\right\},\left\{\xi\left(C^{\prime}\right)_{i}\right\}$ and $\left\{\xi\left(C^{\prime \prime}\right)_{i}\right\}$ and replacing $(m, P)$ by an equivalent pair, we can reduce ourselves to the situation where $P\left(C, C^{\prime}\right)$ is an upper triangular matrix and $P\left(C^{\prime}, C^{\prime \prime}\right)=I$.

Proof. By Lemma 2.2, $P\left(C, C^{\prime}\right)$ is reduced to an upper triangular matrix. In this case, $\left\{\xi\left(C^{\prime}\right)_{1}, \cdots, \xi\left(C^{\prime}\right)_{r}\right\}$ is only rearranged. If we rearrange $\left\{\xi\left(C^{\prime \prime}\right)_{1}, \cdots, \xi\left(C^{\prime \prime}\right)_{r}\right\}$ exactly as $\left\{\xi\left(C^{\prime}\right)_{1}, \cdots, \xi\left(C^{\prime}\right)_{r}\right\}$ is rearranged, then $P\left(C^{\prime}, C^{\prime \prime}\right)$ remains the identity matrix.

Lemma 2.6. Let $C$ and $C^{\prime}$ be two cones in $\Delta(n)$. Suppose that $P=$ $P\left(C, C^{\prime}\right)$ is an upper triangular matrix. Let $\varphi$ be in $|C| \cap\left|C^{\prime}\right|$. Then, by rearranging $\left\{\xi(C)_{i}\right\}$ and $\left\{\xi\left(C^{\prime}\right)_{i}\right\}$, we may assume that $P\left(C, C^{\prime}\right)$ is an upper triangular matrix and that

$$
\varphi\left(\xi(C)_{1}\right) \geq \varphi\left(\xi(C)_{2}\right) \geq \cdots \geq \varphi\left(\xi(C)_{r}\right)
$$

and

$$
\varphi\left(\xi\left(C^{\prime}\right)_{1}\right) \geq \varphi\left(\xi\left(C^{\prime}\right)_{2}\right) \geq \cdots \geq \varphi\left(\xi\left(C^{\prime}\right)_{r}\right)
$$

hold.

Proof. Suppose $\varphi\left(\xi(C)_{h}\right)<\varphi\left(\xi(C)_{h+1}\right)$. Since $\varphi\left(\xi(C)_{i}\right)=\varphi\left(\xi\left(C^{\prime}\right)_{i}\right)$ for every $i$ by Lemma 2.1, we have $P_{h, h+1}=0$. Hence we have $P_{h, h} \neq 0$, $P_{h+1, h+1} \neq 0, P_{h, h+1}=0, P_{h+1, h}=0$. By interchanging the order of $\xi(C)_{h}$ and $\xi(C)_{h+1}$ as well as $\xi\left(C^{\prime}\right)_{h}$ and $\xi\left(C^{\prime}\right)_{h+1}$, we have $\varphi\left(\xi(C)_{h}\right)>\varphi\left(\xi(C)_{h+1}\right)$. After a finite repetition of this process we are done. 
Corollary 2.7. Let $C, C^{\prime}$ be two cones in $\Delta(n)$. Suppose that $P\left(C, C^{\prime}\right)$ is an upper triangular matrix. Let $\varphi_{1}, \cdots, \varphi_{l}$ be elements in $|C| \cap\left|C^{\prime}\right|$. Then, by rearranging $\left\{\xi(C)_{i}\right\}$ and $\left\{\xi\left(C^{\prime}\right)_{i}\right\}$, we may assume that $P\left(C, C^{\prime}\right)$ is an upper triangular matrix and that for every pair of $h$ and $k(h>k)$, one of the following conditions holds:

(a) $\varphi_{i}\left(\xi(C)_{k}\right)=\varphi_{i}\left(\xi(C)_{k+1}\right)=\cdots=\varphi_{i}\left(\xi(C)_{h}\right) \quad$ for $1 \leq i \leq l$.

(b) There exists $v(\leq l)$ such that

$$
\varphi_{i}\left(\xi(C)_{k}\right)=\varphi_{i}\left(\xi(C)_{k+1}\right)=\cdots=\varphi_{i}\left(\xi(C)_{h}\right) \quad \text { for } 1 \leq i<v
$$

and

$$
\varphi_{v}\left(\xi(C)_{k}\right)>\varphi_{v}\left(\xi(C)_{h}\right)
$$

Proof. We first apply Lemma 2.6 to $\varphi_{1}$. If $\varphi_{1}\left(\xi(C)_{k}\right)=\cdots=\varphi_{1}\left(\xi(C)_{h}\right)$, then we further apply Lemma 2.6 to $\varphi_{2}$ with respect to $\left\{\xi(C)_{k}, \cdots, \xi(C)_{h}\right\}$ only. Repeating this procedure, we are done.

\section{§3. The case of $\boldsymbol{P}^{n}$}

From this section on, we restrict ourselves to the case $X=\boldsymbol{P}^{n}$ and consider a $T$-linearized vector bundle $E=E(m, P)$ of rank $r(r \geq 2)$ on $\boldsymbol{P}^{n}$. When $n=1$, a vector bundle on $\boldsymbol{P}^{1}$ is split. Hence we assume $n \geq 2$. Let $\left\{\varphi_{1}, \cdots, \varphi_{n}\right\}$ be a $Z$-base of $N$ and let $\varphi_{0}=-\varphi_{1}-\varphi_{2}-\cdots-\varphi_{n}$. Let $\Delta$ be the fan defined by $\left\{\varphi_{0}, \varphi_{1}, \cdots, \varphi_{n}\right\}$, i.e., $\Delta(n)$ consists of $C_{i}=\sum_{j \neq i} \boldsymbol{R}_{0} \varphi_{j}$ $(i=0,1, \cdots, n)$. Then $\boldsymbol{P}^{n}=T_{N} \operatorname{emb}(\Delta)$ and $U_{C_{i}}=\left\{X_{i} \neq 0\right\}$ is the affine open set in $\boldsymbol{P}^{n}$ corresponding to $C_{i}$, where $X_{0}, \cdots, X_{n}$ are homogeneous coordinates. In this case we note that $C \cap C^{\prime}$ is an $(n-1)$-dimensional cone in $\Delta$ for every pair of cones $C$ and $C^{\prime}$ in $\Delta(n)$.

Proposition 3.1. Suppose that $P\left(C^{\prime}, C^{\prime \prime}\right)=I$ is the identity matrix for some $C^{\prime}$ and $C^{\prime \prime}$ in $\Delta(n)$ with $C^{\prime} \neq C^{\prime \prime}$. Then the T-linearized vector bundle $E$ is a direct sum of T-linearized line bundles, hence, in particular, decomposable.

Proof. Let $C$ be another cone in $\Delta(n)$. By Lemma 2.5, we may assume that $P=P\left(C, C^{\prime}\right)$ is an upper triangular matrix and $P\left(C^{\prime}, C^{\prime \prime}\right)=I$. We show that $P$ can be reduced to the identity matrix. Suppose $P_{h k} \neq 0$ for some $h$ and $k(h<k)$. Then $\varphi\left(\xi(C)_{h}\right) \geq \varphi\left(\xi\left(C^{\prime}\right)_{k}\right)$ for every $\varphi$ in $|C| \cap C^{\prime} \mid$. while $\varphi\left(\xi(C)_{k}\right)=\varphi\left(\xi\left(C^{\prime}\right)_{k}\right)$ by Lemma 2.1. Hence we have $\varphi\left(\xi(C)_{h}\right) \geq \varphi\left(\xi(C)_{k}\right)$ for every $\varphi$ in $|C| \cap\left|C^{\prime}\right|$. 
Put $\{\psi\}=|C|-|C| \cap\left|C^{\prime}\right|$. Then $\psi$ is in $|C| \cap\left|C^{\prime \prime}\right|$ by the definition of $\Delta$. Assume now that $\psi\left(\xi(C)_{h}\right)<\psi\left(\xi(C)_{k}\right)$. Since $P\left(C, C^{\prime \prime}\right)=P\left(C, C^{\prime}\right)$ $P\left(C^{\prime}, C^{\prime \prime}\right)=P$ we have $P\left(C, C^{\prime \prime}\right)_{h k}=P_{h k} \neq 0$, and $\varphi\left(\xi(C)_{h}\right) \geq \varphi\left(\xi\left(C^{\prime \prime}\right)_{k}\right)$ for every $\varphi$ in $|C| \cap\left|C^{\prime \prime}\right|$. Therefore, since $\psi$ is in $|C| \cap\left|C^{\prime \prime}\right|$, we have $\psi\left(\xi(C)_{k}\right)$ $>\psi\left(\xi(C)_{h}\right) \geq \psi\left(\xi\left(C^{\prime \prime}\right)_{k}\right)$. This is a contradiction to Lemma 2.1 since $P\left(C, C^{\prime \prime}\right)=P$ is an upper triangular matrix and $\operatorname{det}(P) \neq 0$. So we have $\psi\left(\xi(C)_{h}\right) \geq \psi\left(\xi(C)_{k}\right)$. Since $|C|=\left(|C| \cap\left|C^{\prime}\right|\right) \cup\{\psi\}$ we have $\varphi\left(\xi(C)_{h}\right) \geq \varphi\left(\xi(C)_{k}\right)$ for every $\varphi$ in $|C|$. Now we define $\sigma(C)=\left(\sigma(C)_{i j}\right)$ by

$$
\sigma(C)_{i j}= \begin{cases}1 & \text { for } i=j, \\ c \neq 0 & \text { for } i=h \text { and } j=k, \\ 0 & \text { otherwise, }\end{cases}
$$

and replace $(m, P)$ by an equivalent pair using this $\sigma(C)$. Then we can reduce ourselves to the case $P\left(C, C^{\prime}\right)_{h k}=0$. If this process is repeated for all $P\left(C, C^{\prime}\right)_{h k} \neq 0(h \neq k)$, then finally $P\left(C, C^{\prime}\right)$ will become a diagonal matrix. By taking $\sigma^{\prime}(C)=\left(\sigma^{\prime}(C)_{i j}\right)$ with $\sigma^{\prime}(C)_{i j}=P\left(C, C^{\prime}\right)_{i j}$ and replacing $(m, P)$ by an equivalent pair using this $\sigma^{\prime}(C)$, we may assume $P\left(C, C^{\prime}\right)=I$. Hence $P\left(C, C^{\prime \prime}\right)=I$.

Furthermore, if $n \geq 3$, let $C^{*}$ be another cone in $\Delta(n)$. We apply the same process to $P\left(C^{*}, C^{\prime}\right)$. Then we may assume $P\left(C^{*}, C^{\prime}\right)=I$. Hence

$$
P\left(C, C^{*}\right)=P\left(C, C^{\prime}\right) P\left(C^{*}, C^{\prime}\right)^{-1}=I .
$$

Therefore all $P\left(C, C^{*}\right)$ can be reduced to the identity matrix. This means that the $T$-linearized vector bundle $E$ is a direct sum of $T$-linearized line bundles by the very construction of $E(m, P)$.

Proposition 3.2. Suppose that $P\left(C, C^{\prime}\right), P\left(C^{\prime}, C^{\prime \prime}\right)$ and $P\left(C^{\prime \prime}, C\right)$ are upper triangular matrices for some triple of pairwise distinct cones $C, C^{\prime}$ and $C^{\prime \prime}$ in $\Delta(n)$. Then the T-linearized vector bundle $E$ is a direct sum of T-linearized line bundles.

Proof. Concerning the first row of $P$ for $(m, P)$, we suppose that there exists $s>1$ such that

$$
P\left(C, C^{\prime}\right)_{1 j}=0, \quad P\left(C^{\prime}, C^{\prime \prime}\right)_{1 j}=0, \quad P\left(C^{\prime \prime}, C\right)_{1 j}=0 \quad \text { for } 1<j<s
$$

and that

$$
P\left(C, C^{\prime}\right)_{1 s} \neq 0, \quad P\left(C^{\prime \prime}, C\right)_{1 s} \neq 0 .
$$

Then, since $P\left(C, C^{\prime}\right)_{1 s} \neq 0$ and $P\left(C^{\prime \prime}, C\right)_{1 s} \neq 0$, we have 


$$
\varphi\left(\xi(C)_{1}\right) \geq \varphi\left(\xi\left(C^{\prime}\right)_{s}\right) \quad \text { for every } \varphi \text { in }|C| \cap\left|C^{\prime}\right|
$$

and

$$
\varphi\left(\xi\left(C^{\prime \prime}\right)_{1}\right) \geq \varphi\left(\xi(C)_{s}\right) \quad \text { for every } \varphi \text { in }\left|C^{\prime \prime}\right| \cap|C| .
$$

Since, by Lemma 2.1,

$$
\varphi\left(\xi(C)_{s}\right)=\varphi\left(\xi\left(C^{\prime}\right)_{s}\right) \quad \text { for every } \varphi \text { in }|C| \cap\left|C^{\prime}\right|
$$

and

$$
\varphi\left(\xi(C)_{1}\right)=\varphi\left(\xi\left(C^{\prime \prime}\right)_{1}\right) \quad \text { for every } \varphi \text { in }\left|C^{\prime \prime}\right| \cap|C|
$$

we have

$$
\varphi\left(\xi(C)_{1}\right) \geq \varphi\left(\xi(C)_{s}\right) \quad \text { for every } \varphi \text { in }|C| \cap\left|C^{\prime}\right|
$$

and

$$
\varphi\left(\xi(C)_{1}\right) \geq \varphi\left(\xi(C)_{s}\right) \quad \text { for every } \varphi \text { in }\left|C^{\prime \prime}\right| \cap|C| .
$$

Since $|C|=\left(|C| \cap\left|C^{\prime}\right|\right) \cup\left(|C| \cap\left|C^{\prime \prime}\right|\right)$ we have

$$
\varphi\left(\xi(C)_{1}\right) \geq \varphi\left(\xi(C)_{s}\right) \quad \text { for every } \varphi \text { in }|C| .
$$

We define $\sigma(C)=\left(\sigma(C)_{i j}\right)$ by

$$
\sigma(C)_{i j}= \begin{cases}1 & \text { for } i=j, \\ c \neq 0 & \text { for } i=1 \text { and } j=s, \\ 0 & \text { otherwise, }\end{cases}
$$

and replace $(m, P)$ by an equivalent pair using this $\sigma(C)$. Then we can reduce ourselves to the case $P\left(C, C^{\prime}\right)_{1 s}=0$. Furthermore if $P\left(C^{\prime \prime}, C\right)_{1 s} \neq 0$ or $P\left(C^{\prime}, C^{\prime \prime}\right)_{1 s} \neq 0$ we do the same. Then we are reduced to the case $P\left(C^{\prime \prime}, C\right)_{1 s}=0$, hence $P\left(C^{\prime}, C^{\prime \prime}\right)_{1 s}=0$. Thus we may assume that

$$
P\left(C, C^{\prime}\right)_{1 j}=0, \quad P\left(C^{\prime}, C^{\prime \prime}\right)_{1 j}=0, \quad P\left(C^{\prime \prime}, C\right)_{1 j}=0 \quad \text { for } j \neq 1 .
$$

Repaet the same process to the other rows successively. Then $P\left(C, C^{\prime}\right)$, $P\left(C^{\prime}, C^{\prime \prime}\right)$ and $P\left(C^{\prime \prime}, C\right)$ become diagonal matrices. We define $\sigma(C)=$ $\left(\sigma(C)_{i j}\right)$ by $\sigma(C)_{i j}=P\left(C, C^{\prime}\right)_{i j}$ and replace $(m, P)$ by an equivalent pair using this $\sigma(C)$. Then we are reduced to the case $P\left(C, C^{\prime}\right)=I$. Therefore, by Proposition 3.1, the $T$-linearized vector bundle $E$ is a direct sum of $T$-linearized line bundles.

Corollary 3.3. Suppose that $P\left(C, C^{\prime}\right)$ and $P\left(C^{\prime}, C^{\prime \prime}\right)$ are upper triangular martices for some triple of pairwise distinct cones $C, C^{\prime}$ and $C^{\prime \prime}$ in 
$\Delta(n)$. Then the T-linearized vector bundle $E$ is a direct sum of T-linearized line bundles.

Theorem 3.4. Let $r>1$ and $n \geq 2$. For a pair $(m, P)$, suppose the corresponding T-linearized vector bundle $E=E(m, P)$ on $P^{n}$ of rank $r$ is indecomposable. Put $|C|=\left\{\varphi_{1}, \cdots, \varphi_{n}\right\}$ for a cone $C$ in $\Delta(n)$. Then, for any pair of distinct integers $s$ and $t(1 \leq s, t \leq n)$, there exist two integers $h$ and $k$ such that

$$
\varphi_{s}\left(\xi(C)_{h}\right)<\varphi_{s}\left(\xi(C)_{k}\right), \quad \varphi_{t}\left(\xi(C)_{h}\right)>\varphi_{t}\left(\xi(C)_{k}\right)
$$

and

$$
\varphi_{i}\left(\xi(C)_{h}\right)=\varphi_{i}\left(\xi(C)_{k}\right) \quad \text { for } i \neq s, t .
$$

Proof. We prove the assertion only when $s=1$ and $t=2$ since the proof in general is the same. Since we are working on $\boldsymbol{P}^{n}$, there exist $C^{\prime}$ and $C^{\prime \prime}$ in $\Delta(n)$ such that

$$
\left|C^{\prime}\right|=\left\{\varphi_{0}, \varphi_{2}, \varphi_{3}, \cdots, \varphi_{n}\right\}, \quad\left|C^{\prime \prime}\right|=\left\{\varphi_{0}, \varphi_{1}, \varphi_{3}, \cdots, \varphi_{n}\right\}
$$

for some $\varphi_{0} \in N$. By Lemma 2.3, we assume that $P\left(C, C^{\prime}\right)_{i i} \neq 0$ for every $i$ and that $P\left(C^{\prime}, C^{\prime \prime}\right)$ is an upper triangular matrix. Hence by Lemma 2.1, we get

$$
\varphi\left(\xi(C)_{i}\right)=\varphi\left(\xi\left(C^{\prime}\right)_{i}\right) \quad \text { for every } \varphi \text { in }|C| \cap\left|C^{\prime}\right|=\left\{\varphi_{2}, \varphi_{3}, \cdots, \varphi_{n}\right\}
$$

and

$$
\varphi\left(\xi\left(C^{\prime}\right)_{i}\right)=\varphi\left(\xi\left(C^{\prime \prime}\right)_{i}\right) \quad \text { for every } \varphi \text { in }\left|C^{\prime}\right| \cap\left|C^{\prime \prime}\right|=\left\{\varphi_{1}, \varphi_{3}, \cdots, \varphi_{n}\right\} .
$$

Therefore

$$
\varphi\left(\xi(C)_{i}\right)=\varphi\left(\xi\left(C^{\prime}\right)_{i}\right)=\varphi\left(\xi\left(C^{\prime \prime}\right)_{i}\right)
$$

for every $\varphi$ in $|C| \cap\left|C^{\prime}\right| \cap\left|C^{\prime \prime}\right|=\left\{\varphi_{3}, \varphi_{4}, \cdots, \varphi_{n}\right\}$. If $n=2$ then this amounts to nothing since $|C| \cap\left|C^{\prime}\right| \cap\left|C^{\prime \prime}\right|=\varnothing$. When $n \geq 3$, we further apply Corollary 2.7 to $\varphi_{3}, \cdots, \varphi_{n}$ and $P\left(C^{\prime}, C^{\prime \prime}\right)$ and may assume that $P\left(C^{\prime}, C^{\prime \prime}\right)$ is an upper triangular matrix and that for every pair $h$ and $k(h>k)$, one of the following conditions holds:

(a) $\varphi_{i}\left(\xi(C)_{k}\right)=\varphi_{i}\left(\xi(C)_{k+1}\right)=\cdots=\varphi_{i}\left(\xi(C)_{h}\right)$ for $3 \leq i \leq n$.

(b) There exists $v$ such that

$$
\varphi_{i}\left(\xi(C)_{k}\right)=\varphi_{i}\left(\xi(C)_{k+1}\right)=\cdots=\varphi_{i}\left(\xi(C)_{h}\right) \quad \text { for } 3 \leq i<v
$$

and 


$$
\varphi_{v}\left(\xi(C)_{k}\right)>\varphi_{v}\left(\xi(C)_{h}\right) .
$$

$P\left(C, C^{\prime}\right)$ cannot be reduced to an upper triangular matrix, since, otherwise, $E$ would be decomposable by Corollary 3.3 contradicting our assumption. Thus there exist integers $h>k$ such that $P\left(C, C^{\prime}\right)_{h k} \neq 0$. Then we have $\varphi\left(\xi(C)_{h}\right) \geq \varphi\left(\xi\left(C^{\prime}\right)_{k}\right)$ for every $\varphi$ in $|C| \cap\left|C^{\prime}\right|$. Since $\varphi\left(\xi(C)_{k}\right)$ $=\varphi\left(\xi\left(C^{\prime}\right)_{k}\right)$ for every $\varphi$ in $|C| \cap\left|C^{\prime}\right|$, we have $\varphi\left(\xi(C)_{h}\right) \geq \varphi\left(\xi(C)_{k}\right)$ for every $\varphi$ in $|C| \cap\left|C^{\prime}\right|$, hence for every $\varphi$ in $\left\{\varphi_{3}, \cdots, \varphi_{n}\right\}=|C| \cap\left|C^{\prime}\right| \cap\left|C^{\prime \prime}\right|$. This means, by (a) and (b) above, that

$$
\varphi\left(\xi(C)_{k}\right)=\varphi\left(\xi(C)_{k+1}\right)=\cdots=\varphi\left(\xi(C)_{h}\right)
$$

for every $\varphi$ in $\left\{\varphi_{3}, \cdots, \varphi_{n}\right\}$. Since $\varphi_{2}$ is in $|C| \cap\left|C^{\prime}\right|$, we have $\varphi_{2}\left(\xi(C)_{h}\right) \geq$ $\varphi_{2}\left(\xi(C)_{k}\right)$ as we saw above. Hence we have the following four possibilities:

1. $\varphi_{1}\left(\xi(C)_{h}\right) \geq \varphi_{1}\left(\xi(C)_{k}\right)$ and $\varphi_{2}\left(\xi(C)_{h}\right)=\varphi_{2}\left(\xi(C)_{k}\right)$,

2. $\varphi_{1}\left(\xi(C)_{h}\right)<\varphi_{1}\left(\xi(C)_{k}\right)$ and $\varphi_{2}\left(\xi(C)_{h}\right)=\varphi_{2}\left(\xi(C)_{k}\right)$,

3. $\varphi_{1}\left(\xi(C)_{h}\right) \geq \varphi_{1}\left(\xi(C)_{k}\right)$ and $\varphi_{2}\left(\xi(C)_{h}\right)>\varphi_{2}\left(\xi(C)_{k}\right)$,

4. $\varphi_{1}\left(\xi(C)_{h}\right)<\varphi_{1}\left(\xi(C)_{k}\right)$ and $\varphi_{2}\left(\xi(C)_{h}\right)>\varphi_{2}\left(\xi(C)_{k}\right)$.

We now show that the case 4 happens for some $h$ and $k(h>k)$ such that $P\left(C, C^{\prime}\right)_{h k} \neq 0$. Suppose that the case 4 does not happen for any such $h, k$. Then, by interchanging $\xi(C)_{h}$ and $\xi(C)_{k}$ if the case 2 happens, we have

$$
\varphi\left(\xi(C)_{h}\right) \geq \varphi\left(\xi(C)_{k}\right) \quad \text { for every } \varphi \text { in }|C| .
$$

Now we take the smallest $k$ such that $P\left(C, C^{\prime}\right)_{h k} \neq 0$ for some $h>k$. We define $\sigma(C)=\left(\sigma(C)_{i j}\right)$ by

$$
\sigma(C)_{i j}= \begin{cases}1 & \text { for } i=j, \\ c \neq 0 & \text { for } i=h \text { and } j=k, \\ 0 & \text { otherwise }\end{cases}
$$

and replace $(m, P)$ by an equivalent pair using this $\sigma(C)$. Then we can reduce ourselves to the case $P\left(C, C^{\prime}\right)_{h k}=0$. Repeating the same procedure for every $h$ such that $h>k$ and $P\left(C, C^{\prime}\right)_{h k} \neq 0$, we have $P\left(C, C^{\prime}\right)_{i k}$ $=0$ for all $i>k$. After this procedure, $P\left(C, C^{\prime}\right)_{i i}$ may be zero for some $i$, but, by rearranging the order of $\left\{\hat{\xi}(C)_{k+1}, \cdots, \xi(C)_{r}\right\}$, we have $P\left(C, C^{\prime}\right)_{i i}$ $\neq 0$ for all $i>k$. So we can successively apply the same procedure, and $P\left(C, C^{\prime}\right)$ is finally reduced to an upper triangular matrix. By Corollary 3.3 , this is a contradiction to the indecomposability of $E$. Therefore there exist $h$ and $k$ such that 


$$
\varphi_{1}\left(\xi(C)_{h}\right)<\varphi_{1}\left(\xi(C)_{k}\right), \quad \varphi_{2}\left(\xi(C)_{h}\right)>\varphi_{2}\left(\xi(C)_{k}\right)
$$

and

$$
\varphi_{i}\left(\xi(C)_{h}\right)=\varphi_{i}\left(\xi(C)_{k}\right) \quad \text { for } i \geq 3 .
$$

Corollary 3.5. Suppose $1<r \leq n(n \geq 2)$ and let $E$ be an indecomposable T-linearized vector bundle of rank $r$ on $\boldsymbol{P}^{n}$. Then we have:

(1) $r=n$.

(2) For every $C \in \Delta(n)$ and every $\varphi$ in $|C|$, all except one of $\left\{\varphi\left(\xi(C)_{1}\right)\right.$, $\left.\cdots, \varphi\left(\xi(C)_{n}\right)\right\}$ are the same integers.

(3) Let $C=\boldsymbol{R}_{0} \varphi_{1}+\cdots+\boldsymbol{R}_{0} \varphi_{n}$ be in $\Delta(n)$. We can tensor a suitable $T$-linearized line bundle to $E$ and rearrange the order of $\left\{\xi(C)_{1}, \cdots, \xi(C)_{n}\right\}$, so that the following hold for every $i=1, \cdots, n$ :

$$
\varphi_{i}\left(\xi(C)_{i}\right)=a_{i} \text { and } \varphi_{i}\left(\xi(C)_{j}\right)=0 \quad \text { for any } j \neq i \text {. }
$$

In this case, $a_{1}, \cdots, a_{n}$ are all positive or all negative.

Proof. Let $|C|=\left\{\varphi_{1}, \cdots, \varphi_{n}\right\}$ for a $C \in \Delta(n)$, and apply Theorem 3.4 to $C$. For each $s$, we first see that $\varphi_{s}\left(\xi(C)_{1}\right), \cdots, \varphi_{s}\left(\xi(C)_{r}\right)$ cannot be all equal, since we can pick $t \neq s$ and apply Theorem 3.4 to the pair $(s, t)$.

Clearly, Theorem 3.4 gives a one-to-one map from the set $\{(s, t) \mid 1 \leq s$ $<t \leq n\}$ to the set of pairs $\{h, k\}$ of distinct integers between 1 and $r$. Thus $n(n-1) / 2 \leq r(r-1) / 2$. Since $r \leq n$ by assumption, we have $r=n$, and the above map must be a bijection.

We can so rearrange $\xi(C)_{1}, \cdots, \xi(C)_{n}$ that for each $i$, the pair $(1, i)$ is sent to the pair $\{1, i\}$ by the above map. Then for each $i$, we see that $\varphi_{i}\left(\xi(C)_{j}\right)$ are equal for all $j \neq i$.

In view of Remark 1.5, we may tensor a $T$-linearized line bundle to $E$ so that the following holds for each $i$ :

$$
\varphi_{i}\left(\xi(C)_{i}\right)=a_{i} \text { and } \varphi_{i}\left(\xi(C)_{j}\right)=0 \quad \text { for any } j \neq i .
$$

By Theorem 3.4, we see that $a_{s}, a_{t}$ should have the same sign for all $s \neq t$.

\section{$\S 4$. Determination of $P\left(C, C^{\prime}\right)$}

In this section we consider $P\left(C, C^{\prime}\right)$ for an indecomposable $T$-linearized vector bundle of rank $n$ on $\boldsymbol{P}^{n}(n \geq 2)$. By Corollary 3.5, we may assume that, for every $C$ and every $\varphi$ in $|C|$, we have $\varphi\left(\xi(C)_{i}\right) \geq 0$ and $\varphi\left(\xi(C)_{i}\right)=0$ except for one $i$. For $C, C^{\prime}, C^{\prime \prime} \in \Delta(n)$, let 


$$
\begin{aligned}
|C| & =\left\{\varphi_{1}, \varphi_{2}, \varphi_{3}, \cdots, \varphi_{n}\right\}, \\
\left|C^{\prime}\right| & =\left\{\varphi_{0}, \varphi_{2}, \varphi_{3}, \cdots, \varphi_{n}\right\}, \\
\left|C^{\prime \prime}\right| & =\left\{\varphi_{0}, \varphi_{1}, \varphi_{3}, \cdots, \varphi_{n}\right\} .
\end{aligned}
$$

By changing the order of $\left\{\xi(C)_{i}\right\},\left\{\xi\left(C^{\prime}\right)_{i}\right\}$ and $\left\{\xi\left(C^{\prime \prime}\right)_{i}\right\}$, we assume that

$$
\begin{array}{rlr}
\left(\varphi_{0}\left(\xi\left(C^{\prime}\right)_{1}\right), \cdots, \varphi_{0}\left(\xi\left(C^{\prime}\right)_{n}\right)\right) & =\left(\varphi_{0}\left(\xi\left(C^{\prime \prime}\right)_{1}\right), \cdots, \varphi_{0}\left(\xi\left(C^{\prime \prime}\right)_{n}\right)\right) \\
& =(a, 0,0, \cdots, 0) \quad(a>0), \\
\left(\varphi_{1}\left(\xi(C)_{1}\right), \cdots, \varphi_{1}\left(\xi(C)_{n}\right)\right) & =(b, 0,0, \cdots, 0), \\
\left(\varphi_{1}\left(\xi\left(C^{\prime \prime}\right)_{1}\right), \cdots, \varphi_{1}\left(\xi\left(C^{\prime \prime}\right)_{n}\right)\right) & =(0, b, 0, \cdots, 0) \quad(b>0), \\
\left(\varphi_{2}\left(\xi(C)_{1}\right), \cdots, \varphi_{2}\left(\xi(C)_{n}\right)\right) & =\left(\varphi_{2}\left(\xi\left(C^{\prime}\right)_{1}\right), \cdots, \varphi_{2}\left(\xi\left(C^{\prime}\right)_{n}\right)\right) \\
& =(0, c, 0, \cdots, 0) \quad(c>0),
\end{array}
$$

and

$$
\begin{aligned}
\left(\varphi_{i}\left(\xi(C)_{1}\right), \cdots, \varphi_{i}\left(\xi(C)_{n}\right)\right) & =\left(\varphi_{i}\left(\xi\left(C^{\prime}\right)_{1}\right), \cdots, \varphi_{i}\left(\xi\left(C^{\prime}\right)_{n}\right)\right) \\
& =\left(\varphi_{i}\left(\xi\left(C^{\prime \prime}\right)_{1}\right), \cdots, \varphi_{i}\left(\xi\left(C^{\prime \prime}\right)_{n}\right)\right) \\
& =\left(0, \cdots, 0, d_{i}, \cdots, 0\right)
\end{aligned}
$$

for $i \geq 3$, where $d_{i}>0$ is the $i$-th entry.

Then, by (II), we have

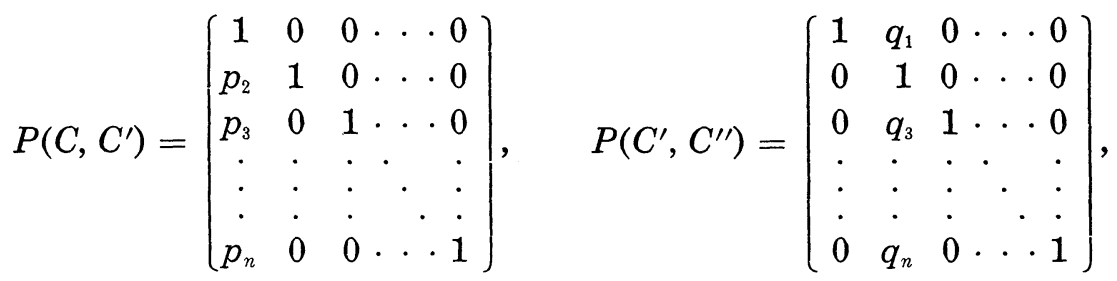

$$
\begin{aligned}
& P\left(C^{\prime}, C\right)=\left(\begin{array}{ccccc}
0 & -1 & 0 & \cdots & 0 \\
1 & r_{2} & 0 & \cdots & 0 \\
0 & r_{3} & 1 & \cdots & 0 \\
\vdots & \vdots & \vdots & \ddots & \vdots \\
\vdots & \vdots & \vdots & \ddots & \vdots \\
0 & r_{n} & 0 & \cdots & 1
\end{array}\right)
\end{aligned}
$$

Since $P\left(C, C^{\prime}\right) P\left(C^{\prime}, C^{\prime \prime}\right) P\left(C^{\prime \prime}, C\right)=I$ we have:

LemMA 4.1. $q_{1}=1, \quad p_{2}=-1, \quad r_{2}=1$ and

$$
p_{i}=-q_{i}=r_{i} \text { for } 3 \leq i \leq n .
$$

Lemma 4.2. $p_{i} \neq 0$ for $2 \leq i \leq n$. 
Proof. Fix two cones $C$ and $C^{\prime}$ and $P\left(C, C^{\prime}\right)$. We take another $C^{\prime \prime}$ successively and calculate in the above way. Thən we have $p_{i} \neq 0$ for $2 \leq i \leq n$.

LEMmA 4.3. We may assume that $p_{i}=1$ for $1<i \leq n$.

Proof. Since $p_{i} \neq 0$ for $i \geq 2$, we take

$$
\sigma(C)=\sigma\left(C^{\prime}\right)=\left\{\begin{array}{lll}
1 & & \\
p_{\Omega} & & \\
0 & & \cdot \\
0 & & p_{n}
\end{array}\right)
$$

and replace $(m, P)$ by an equivalent pair using these $\sigma(C), \sigma\left(C^{\prime}\right)$. Then we have

$$
P\left(C, C^{\prime}\right)=\left(\begin{array}{cccc}
1 & 0 & \cdots & 0 \\
1 & 1 & & 0 \\
\vdots & & . & \\
1 & 0 & & 1
\end{array}\right)
$$

Hence we may assume that $p_{i}=1$ for $1<i \leq n$.

If $P\left(C, C^{\prime}\right)$ is in the above form, then $P\left(C^{\prime}, C^{\prime \prime}\right)$ and $P\left(C^{\prime \prime}, C\right)$ are naturally determined if $m$ in (I) is given. Therefore $P$ in $(m, P)$ is determined for every pair of cones in $\Delta(n)$. Hence, for each indecomposable $T$-linearized vector bundle of $\operatorname{rank} n, P$ is unique up to equivalence (III). Therefore, for any given $m$ in (I) which we know by (3) of Corollary 3.5, an indecomposable $T$-linearized vector bundle is uniquely determined if it exists.

Theorem 4.4. Let $E$ be a T-linearized vector bundle defined by the sequence

$$
0 \longrightarrow \mathcal{O}_{\boldsymbol{P}_{n}} \stackrel{f}{\longrightarrow} \bigoplus_{i=0}^{n} \mathcal{O}_{\boldsymbol{P}_{n}}\left(a_{i}\right) \longrightarrow E \longrightarrow 0
$$

such that $f$ sends 1 to $\left(X_{0}^{a_{0}}, X_{1}^{a_{1}}, \cdots, X_{n}^{a_{n}}\right)$, where $X_{0}, \cdots, X_{n}$ are homogeneous coordinates of $\boldsymbol{P}^{n}$ and $a_{0}, \cdots, a_{n}$ are positive integers. Then $E$ is an indecomposable vector bundle.

Proof. Suppose $E$ is decomposable and let $E=E_{1} \oplus E_{2} \oplus \cdots \oplus E_{l}$ with $l \geq 2$ be a decomposition of $E$ into indecomposable vector bundles. Every indecomposable component $E_{i}$ is $T$-equivariant by virtue of the 
Krull-Schmidt Theorem (see [1]). Since $\operatorname{rank}\left(E_{i}\right)<n, E_{i}$ is necessarily a line bundle by Corollary 3.5. Hence $E$ is a direct sum of line bundles and we may let

$$
E=\mathcal{O}_{\boldsymbol{P}^{n}}\left(d_{1}\right) \oplus \mathcal{O}_{\boldsymbol{P}^{n}}\left(d_{2}\right) \oplus \cdots \oplus \mathcal{O}_{\boldsymbol{P}^{n}}\left(d_{n}\right)
$$

for $d_{1} \leq d_{2} \leq \cdots \leq d_{n}$. We may assume that $a_{0} \leq a_{1} \leq \cdots \leq a_{n}$. By tensoring the sequence $(*)$ with $\mathcal{O}_{P^{n}}(-k)$ for $k>0$ we have

$$
h^{0}\left(\bigoplus_{i=0}^{n} \mathcal{O}_{\boldsymbol{P}^{n}}\left(a_{i}-k\right)\right)=h_{i}{ }^{n}(E(-k)) .
$$

We have a contradiction, if we take $k=a_{n}$ when $a_{n}>d_{n}$ while we take $k=d_{n}$ when $a_{n}<d_{n}$. Hence we have $a_{n}=d_{n}$. Similarly, we have $a_{i}=d_{i}$ for $1 \leq i \leq n$. By $(*)$, we have $\operatorname{det}(E)=\mathcal{O}_{P_{n}}\left(\sum_{i=0}^{n} a_{i}\right)$, which is equal to $\mathcal{O}_{P^{n}}\left(\sum_{i=1}^{n} d_{i}\right)$. Hence $a_{0}=0$ and $(*)$ is split, a contradiction. Therefore $E$ is indecomposable.

If we take $a_{0}=a_{1}=\cdots=a_{n}=1$ in Theorem 4.4, then the $T$-linearized vector bundle $E$ is the tangent bundle $T_{\boldsymbol{P}^{n}}$ for $\boldsymbol{P}^{n}$.

Corollary 4.5. $T_{\boldsymbol{P}^{n}}$ is indecomposable.

By short calculation, we have

$$
m\left(\varphi_{i}\right)=\left(-a_{i}, 0, \cdots, 0\right) \quad \text { for } 0 \leq i \leq n
$$

for the $T$-linearized vector bundle $E$ which is defined by (*) in Theorem 4.4. Therefore we have:

THEOREM 4.6. An indecomposable equivariant vector bundle of rank $n$ on $\boldsymbol{P}^{n}(n \geq 2)$ is isomorphic to $E(d)$ or $E^{*}(d)$ for some integer $d$, where $E$ is defined by the sequence $(*)$ in Theorem 4.4 for some positive integers $a_{i}$ $(0 \leq i \leq n)$.

\section{REFERENCES}

[1] M. F. Atiyah, On the Krull-Schmidt Theorem with applications to sheaves, Bull. Soc. Math. France, 84 (1956), 307-317.

[2] J. Bertin et G. Elencwajg, Symétries des fibrés vectorieles sur $\boldsymbol{P}^{n}$ et nombre d'Euler, Duke Math. J., 49 (1982), 807-831.

[ 3 ] R. Hartshorne, Stable vector bundles of rank 2 on $\boldsymbol{P}^{3}$, Math. Ann., 238 (1978), 229-280.

[4] T. Kaneyama, On equivariant vector bundles on an almost homogeneous variety, Nagoya Math. J., 57 (1975), 65-86.

[5] Kempf and Mumford, Toroidal embeddings, Springer Lecture notes 339. 
[6] T. Oda, Torus embeddings and applications, Tata Institute of Fundamental Research 58, (1978).

[ 7 ] T. Oda and K. Miyake, Almost homogeneous algebraic varieties under torus action, Manifolds Tokyo 1973, 373-381, Proceedings of International Conference on Manifolds and Related Topics in Topology.

Department of Mathematics

Gifu College of Education

Yanaizu-cho Hashima-gun

Gifu 501-61, Japan 